\title{
dS vacua and the swampland
}

\author{
Renata Kallosh, ${ }^{a}$ Andrei Linde, ${ }^{a}$ Evan McDonough ${ }^{b}$ and Marco Scalisi ${ }^{c}$ \\ ${ }^{a}$ Stanford Institute for Theoretical Physics and Department of Physics, Stanford University, \\ Stanford, CA 94305, U.S.A. \\ ${ }^{b}$ Department of Physics, Brown University, \\ Providence, RI 02903, U.S.A. \\ ${ }^{c}$ Institute for Theoretical Physics, Katholieke Universiteit Leuven, \\ Celestijnenlaan 200D, B-3001 Leuven, Belgium \\ E-mail: kallosh@stanford.edu, alinde@stanford.edu, \\ evan_mcdonough@brown.edu, marco.scalisi@kuleuven.be
}

ABSTRACT: In this note we revisit some of the recent $10 \mathrm{~d}$ and $4 \mathrm{~d}$ arguments suggesting that the uplifting of supersymmetric AdS vacua leads to a flattening of the potential, preventing the formation of dS vacua. We explain why the corresponding 10d approach is inconclusive and requires considerable modifications. We also show that while the flattening effects may occur for some extreme values of the parameters, they do not prevent the formation of $\mathrm{dS}$ vacua within the range of validity of the $4 \mathrm{~d}$ KKLT models. The KL version of the KKLT scenario based on a racetrack superpotential requires parametrically small uplifting, which is not affected by flattening. We show that this scenario is compatible with the weak gravity conjecture for a broad choice of parameters of the KL model. Thus, the results of our analysis do not support the recent swampland conjecture.

KeYwORDS: Superstring Vacua, Supergravity Models

ARXIV EPRINT: 1901.02022 


\section{Contents}

1 Introduction 1

2 Gaugino condensation in 10d 3

$\begin{array}{lll}3 & \text { KKLT model and its consistent generalizations } & 5\end{array}$

$\begin{array}{lll}4 & \text { Flattening effects in KKLT } & 7\end{array}$

5 The KL model and the weak gravity conjecture 9

6 Conclusions 14

\section{Introduction}

The string theory landscape scenario emerged in an attempt to solve two problems simultaneously: to provide a mechanism of de Sitter (dS) vacuum stabilization in string theory, and to solve the cosmological constant problem. One of the most popular versions of this theory is based on the KKLT scenario [1], but other mechanisms of dS vacuum stabilization are also available.

The most important part of $\mathrm{dS}$ constructions in string theory is the enormous multiplicity of vacuum states in the theory and the possibility to tunnel from one of these states to another [1-5]. This allows to use the anthropic constraint $\left|V_{d S}\right| \lesssim 10^{-120}$ to account for the incredible smallness of the vacuum energy: if there were some vacua with the required energy density prior to the calculation of quantum corrections, then many of them may move away from the anthropic range when quantum corrections are taken into account, but many other vacua will enter this range. Thus one should be able to find many suitable vacuum states even though the precise vacuum energy in these states cannot be calculated with the precision $\left|V_{d S}\right| \lesssim 10^{-120}$.

This basic idea makes the string landscape scenario very robust. It cannot be invalidated by arguments of naturalness, by the weak gravity conjecture, or by the possibility that radiative corrections may affect $\mathrm{dS}$ vacua. To disprove this scenario one would have to prove a no-go theorem, that would state that all of the $10^{500}$ or more dS vacua in string theory cannot exist, as suggested recently in the new swampland conjecture [6, 7], which we will call the no-dS conjecture. Despite many attempts to prove such no-go theorem during the last 15 years, no such proof is available [8].

One could argue that even though the new swampland conjecture $[6,7]$ may not have a derivation or proof, it can be validated if future observations demonstrate that dark energy has equation of state different from the dS equation of state $w=-1$. However, this is not the case. First of all, all models of dark energy proposed in [6] are already disfavored 
at a statistical significance higher than $4.5 \sigma[8,9]$. Secondly, the models of dark energy with $w \neq-1$ can be constructed in the string theory landscape scenario with dS vacua. Moreover, the possibility to roll down towards the asymptotic dS state may simplify the construction of quintessence models, see e.g. $[8,10]$ and references therein.

Nevertheless, since this issue is extremely important, one should carefully examine any piece of evidence against the string landscape scenario. One of the main arguments in support of the no-dS conjecture was based on the suggestion of [11] that a certain version of the KKLT scenario does not lead to a consistent uplifting of the supersymmetric AdS vacuum to a metastable dS vacuum.

The investigation performed in [11] consists of two parts: a detailed but very complicated $10 \mathrm{~d}$ analysis, and a $4 \mathrm{~d}$ investigation of the KKLT model, which is modified to take into account backreaction. The basic argument of [11] is that, during the uplifting, the potential flattens because of the backreaction to the uplifting, and dS minimum never forms. The authors effectively propose that the backreaction to the uplifting is many orders of magnitude stronger that the basic effect of uplifting. This is a very unconventional proposition, which should be carefully examined.

Because of the complexity of the 10d investigation, the authors of [11] were forced to make various assumptions, or rely upon results previously obtained in different contexts. In particular, some results of the 10d investigation of gaugino condensation in [11] contain divergences, which required regularization and interpretation based on various conjectures [11]. The original analysis of these divergences in [11] contained an error corrected in [12]. Moreover, a recent examination of this issue in $[13,14]$ suggests that these divergences do not appear at all if one takes into account the 4 -fermion non-derivative coupling, which was neglected in $[11,12]$. We present a short discussion of this issue in section 2. This is just one of the many reasons to believe that the uncertainties involved in the $10 \mathrm{~d}$ investigation of $[11,12]$ make its results inconclusive [8, 15-17].

The $4 \mathrm{~d}$ analysis of the KKLT model in [11] was supposed to confirm the 10d approach and the absence of dS vacua. However, a more detailed investigation in [16, 17] demonstrated that the modified 4d KKLT model proposed in [11] is inconsistent, whereas all known consistent $4 \mathrm{~d}$ versions of the KKLT scenario, in the domain of their validity, do not support the recent swampland conjecture $[6,7]$ and are fully compatible with the existence of dS vacua in string theory.

The results of $[16,17]$ are pretty straightforward and can be easily verified. However, recent comments on these issues in [12] show that a more detailed explanation of the relation between uplifting, flattening, and $\mathrm{dS}$ vacua is warranted. We discuss these issues in sections 3 and 4 , and demonstrate by an explicit example that dS vacua do appear for proper choice of parameters even if one assumes extremely strong backreaction and flattening of the potential.

In section 5 we discuss the KL version of the KKLT mechanism [18]. It is based on a particular version of the racetrack superpotential; it is free from the problems discussed in [11]. Recently Van Riet et al. argued $[12,19]$ that there might be some tension between this class of models and a specific version of the weak gravity conjecture introduced in [19]. However, the particular version of the weak gravity conjecture used in [19] is lacking evi- 
dence, ${ }^{1}$ and the authors provided no suggestions for how one could justify it. Moreover, an investigation of this issue by Blanco-Pillado et al. [20] demonstrated that one can satisfy even the unconventional version of the weak gravity conjecture introduced in [19] by a proper choice of parameters of the KL model. Our investigation of this problem in section 5 confirms and strengthens the results of [20] for a broad set of parameters of the KL model. Thus, this model, which allows strong vacuum stabilization protected by supersymmetry $[8,21,22]$ and is most suitable for constructing realistic inflationary models [23-25], does not seem to suffer from any problems with uplifting and stabilization of dS vacua mentioned in $[11,12,19]$.

\section{Gaugino condensation in 10d}

Gaugino condensation refers to the formation of a non-vanishing fermion condensate $\operatorname{Tr}\langle\lambda \lambda\rangle$ in the theory. It plays a significant role in the KKLT construction of de Sitter vacua, since it provides a non-perturbative part of the superpotential $W_{n p}=A e^{-a T}$, where $T$ is the volume of the 4-cycle wrapped by condensing D7 branes. The fermionic part of the $10 \mathrm{~d}$ analysis of $[11,12]$ relies on earlier papers [26-30], where gaugino condensation was studied at quadratic order in the fermions. Meanwhile, $[11,12]$ deduce from $[26-30]$ the gaugino condensate contribution to the $4 \mathrm{~d}$ effective action at quartic order in the fermions, but neglect the intrinsic 4-gaugino interaction on D7-branes. This latter fact makes the analysis of $[11,12]$ incomplete, as pointed out in [13, 14].

Technically, the computation was performed in [26-30] using the gauge-fixed $\kappa$ symmetric Dp-brane action. It was explicitly stressed there that the result is only valid in the approximation quadratic in fermions. This action is supersymmetric and is known only in the case of a single Dp-brane with an Abelian vector multiplet. The relevant coupling was presented in $[29,30]$,

$$
S_{\mathrm{D} 7}^{\mathrm{Maxw}} \supset-\frac{i}{32 \pi l_{s}^{4}} \int_{X^{4}} d^{4} x \bar{\lambda}_{\dot{\alpha}} \bar{\lambda}^{\dot{\alpha}} \int_{D} G_{3} \cdot \Omega J \wedge J+\text { c.c. }
$$

The D7-brane fills the four-dimensional space $X_{4}$ and wraps the four-cycle D of the internal manifold. Note that here $G_{3}$ couples only to the fermion on a single D7, $\lambda$ does not have a non-Abelian gauge group index.

The next step proposed in [27-30] is that for many coincident D7 branes, the 3 -form couples to the trace of the fermion bilinears on the surface of $N$ coincident D7 branes,

$$
S_{\mathrm{D} 7}^{\mathrm{YM}} \supset-\frac{i}{32 \pi l_{s}^{4}} \int_{X^{4}} d^{4} x \operatorname{Tr}\left(\bar{\lambda}_{\dot{\alpha}} \bar{\lambda}^{\dot{\alpha}}\right) \int_{D} G_{3} \cdot \Omega J \wedge J+c . c .
$$

In [27-30] the issue of the 4-fermion coupling on the surface of the $N$ coincident D7 branes was not studied. Meanwhile, in [11] (see also [12]) an assumption was made that replacing $\bar{\lambda}_{\dot{\alpha}} \bar{\lambda}^{\dot{\alpha}}$ by $\operatorname{Tr} \bar{\lambda}_{\dot{\alpha}} \bar{\lambda}^{\dot{\alpha}}$ when describing many D7 branes is the only generalization required. The authors of [11] evaluated the corresponding 4-fermion terms using $G_{3}$ equations of motion, and found divergences, which required regularization.

\footnotetext{
${ }^{1}$ We are grateful to Westphal, McAllister and Rudelius for emphasizing this point to us.
} 
The issues with the 4 -fermion $10 \mathrm{~d}$ analysis in $[11,12]$ were recently discussed in $[13,14]$. In [13] a proposal was made that the 4-fermion terms should be present on the D7 brane, which might resolve the disagreement with the $4 \mathrm{~d}$ analysis. They suggested to follow the related set up in [31-33] for an M-theory compactified on a one-dimensional interval. The action in [31-33] has a perfect square term

$$
-\int d^{11} x \sqrt{g}\left(G_{\mu \nu \rho 11}-c \delta\left(x_{11}\right) \operatorname{Tr} \lambda \Gamma_{\mu \nu \rho} \lambda\right)^{2}
$$

and the $\delta(0)$ singularities cancel due to the perfect square structure in the action, which requires the presence of the 4 -fermion term. A proposal made in [13] for type IIB theory with coincident compactified D7 branes has additional features, which, in particular, allow to reach a nice correspondence with the 4 -fermion terms in $4 \mathrm{~d}$ action.

In [14] it was stressed that, as shown in $[34,35]$, the 4 d supergravity action has a term with the square of the auxiliary field, $|F|^{2}$. And since the on shell value of the auxiliary field involves gaugino

$$
F^{\alpha}=-e^{K / 2} g^{\alpha \beta} \bar{\nabla}_{\bar{\beta}} \bar{W}+\frac{1}{4} \bar{f}_{A B \bar{\beta}} g^{\bar{\beta} \alpha} \bar{\lambda}^{A} P_{L} \lambda^{B},
$$

one finds that the bilinear and quartic dependence on gaugino's comes via a perfect square in the action, $|F|^{2}$.

It was also pointed out in [14] that the existence of the perfect square term in 10d action is a general feature of the Einstein-Yang-Mills supergravity [36-38] and of the underlying 10d superspace geometry. The complete 10d supergravity action, where we suppress terms depending on gravitino and dilatino but keep all terms with gaugino and bosons, is

$$
\begin{aligned}
e^{-1} \mathcal{L}= & -\frac{1}{2} R-\frac{3}{4} \phi^{-\frac{3}{2}}\left(F_{\mu \nu \rho}^{\mathrm{YM}}-\frac{\sqrt{2}}{24} \phi^{\frac{3}{4}} \operatorname{Tr} \bar{\lambda} \Gamma_{\mu \nu \rho} \lambda\right)^{2} \\
& -\frac{9}{16}\left(\frac{\partial_{\mu} \phi}{\phi}\right)^{2}-\frac{1}{4} \operatorname{Tr}\left(F_{\mu \nu}^{\mathrm{YM}}\right)^{2}-\frac{1}{2} \operatorname{Tr} \bar{\lambda} \gamma^{\mu} D_{\mu} \lambda .
\end{aligned}
$$

Type IIB theory in presence of calibrated Dp-branes and Oq-planes as local sources has only $1 / 2$ of the maximal $10 \mathrm{~d}$ supersymmetry. Therefore the effective action describing IIB supergravity with coincident D9-branes is an action of the Einstein-Yang-Mills supergravity, up to the Born-Infeld type terms with higher derivatives. Therefore gauginos which live on the coincident D9 branes must have a 4 -fermion coupling. Dimensional reduction to coincident D7 branes suggests that they also must have a 4 -fermion coupling.

The reason why this was not discussed in [26-30] is that their derivation of eq. (2.1) was based on the $\kappa$-symmetric Abelian D7-brane action, which is supersymmetric upon gauge-fixing. For the Abelian vector multiplet which lives on the brane the 4 -fermion gaugino interaction vanishes since $\left(\bar{\lambda} \Gamma_{\mu \nu \rho} \lambda\right)^{2}=0$. Therefore, even if in [26-30] the issue of the non-derivative 4-fermion coupling would be raised, the answer would be negative, based on the $\kappa$-symmetric Abelian D7-brane action.

But in $[11,12]$ the dependence on non-Abelian gaugino, derived in [26-30] and shown here in eq. (2.2), was assumed to be valid without any additional gaugino-dependent terms 
even for non-Abelian vector multiplets on D7. From Einstein-Yang-Mills supergravity (2.5) it follows that the bilinear in gaugino terms must be accompanied by the quartic gaugino term. This means that the assumption in $[12,19]$ about the absence of 4 -fermion terms in the action (2.2) cannot be valid, and therefore the whole $10 \mathrm{~d}$ analysis needs to be reconsidered. It is likely that it will reduce to the construction of the kind given in [13], and the consistency with $4 \mathrm{~d}$ physics in this aspect will be restored. In what follows we will concentrate on the $4 \mathrm{~d}$ analysis of the KKLT construction.

\section{$3 \quad$ KKLT model and its consistent generalizations}

The KKLT model in the $4 \mathrm{~d}$ supergravity formulation can be described by a superpotential

$$
W=W_{0}+A e^{-a T}+b S .
$$

Here $S$ in the nilpotent multiplet representing anti-D3 brane responsible for the uplifting [39-41]. The Kähler potential can be either

$$
K=-3 \log (T+\bar{T})+S \bar{S}
$$

or

$$
K=-3 \log (T+\bar{T}-S \bar{S}) .
$$

The modification proposed in [11] introduces an extra term $c A e^{-a T} S$ in the superpotential, with an extra parameter $c$ describing effects of backreaction

$$
W=W_{0}+A e^{-a T}+c A e^{-a T} S+b S .
$$

It was argued in [11] that $|c A| \gg b$. This would imply that the backreaction is much greater than the main effect. This is an unusual proposition which does not seem well motivated $[15,16]$.

Instead of debating the reliability of the assumption $|c A| \gg b$, we studied the general case, including $|c A| \ll b$ as well as $|c A| \gg b$. According to [11], the value of $c$ is exponentially sensitive to various parameters of compactification, so in the string theory landscape it may take many different values for any given $b$. Therefore, following [16, 17], we will analyze the potential as a function of two independent parameters $b$ and $c$, and check whether it may have dS vacua.

After Kähler transformation $\left(c A e^{-a T}+b\right) S \rightarrow S$ the model can be equivalently represented as

$$
\begin{aligned}
& W=W_{0}+A e^{-a T}+S \\
& K=-3 \log \left(T+\bar{T}-\frac{S \bar{S}}{\left|c A e^{-a T}+b\right|^{2}}\right) .
\end{aligned}
$$

The denominator in (3.6) is a perfect square, which is positive everywhere except the point $T_{0}=\frac{1}{a} \ln (-c A / b)$, where it vanishes. As emphasized in $[16,17]$, this makes the use of the nilpotent multiplet $S$ in the model (3.4) inconsistent for $|c A|>b$. (A similar problem 


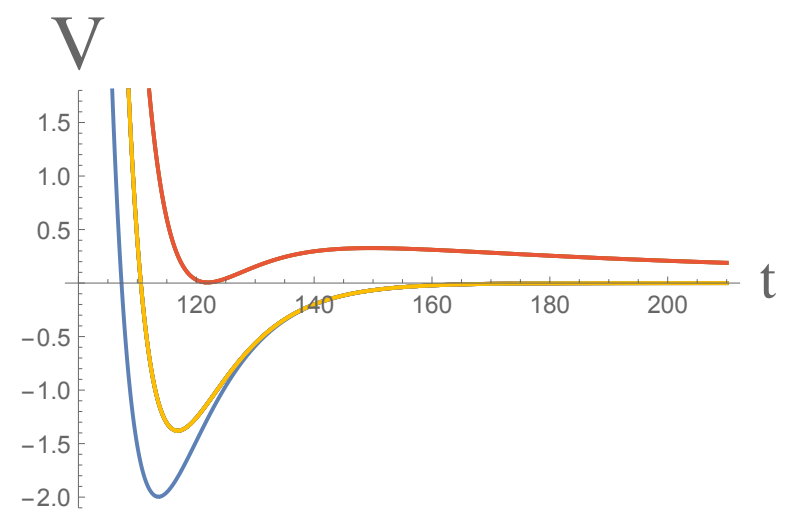

Figure 1. The potential of the theory (3.7) (multiplied by $10^{15}$ ) for $A=1, a=0.1, W_{0}=-10^{-4}$, $0<\beta \ll 1$. The blue (lower) line shows the potential with a supersymmetric AdS minimum prior to uplifting, at $b=c=0$. The second (yellow), line shows the potential at $b=0$ uplifted by increase of $c$ to $c=1$. Finally, the upper (red) line shows the potential with a dS (nearly Minkowski) minimum for $c=1, b=10^{-5}$. The main part of the uplifting is not due to the large change of $c$ from 0 to 1 , but due to the tiny change of $b$ from 0 to $b=10^{-5}$.

appears at large $c$ in the model proposed in [42]; see [17]). The inconsistency disappears for $|c A|<b$, because in that case the point $T_{0}=\frac{1}{a} \ln (-c A / b)$ is outside the physical domain with $\operatorname{Re} \mathrm{T}>0$. In that case, the effects of backreaction are exponentially suppressed, and dS uplifting occurs as in the original model (3.1).

Note that the inconsistency of the $4 \mathrm{~d}$ model proposed in [11] is rather subtle; it manifests itself only if one considers the full supergravity model including fermions. If one temporarily ignores this issue and calculates the bosonic potential, one finds that the theory does contain a large family of dS vacua [16].

To avoid the inconsistency problem altogether, one can add a positive number or function to $\left|c A e^{-a T}+b\right|^{2}$ in (3.6). This removes the pole in the denominator in (3.6), which makes the theory consistent. For example, one may consider the Kähler potential

$$
K=-3 \log \left(T+\bar{T}-\frac{S \bar{S}}{\left|c A e^{-a T}+b\right|^{2}+\beta c^{2} A^{2} e^{-a(T+\bar{T})}}\right)
$$

where $\beta$ is some positive number [17]. This immediately makes $K^{S \bar{S}}$ strictly positive definite, which avoids all inconsistencies of the models of $[11,42]$ for any choice of $\beta>0$.

In particular, for small $\beta$ the potential in the model (3.7) practically coincides with the KKLT potential shown in one of the figures in [16], which we reproduce here for convenience in figure 1. Thus our results about the existence of dS vacua contained in [16] are confirmed for a large range of parameters for consistent generalizations of the KKLT scenario [17]. In particular, for small $\beta$ the potential in the model (3.7) practically coincides with the potential shown in one of the figures in [16], which we reproduce here for convenience. The potential is shown as a function of $t=\operatorname{Re} T$.

Yet another consistent model is described by $W(3.5)$ and Kähler potential

$$
K=-3 \log \left(T+\bar{T}-\frac{S \bar{S}}{|b|^{2}+|c|^{2} e^{-a(T+\bar{T})}}\right),
$$


This model is consistent for any choice of $\{b, c\}$. It also has $\mathrm{dS}$ vacua for a broad choice of its parameters [17].

\section{$4 \quad$ Flattening effects in KKLT}

Recently there was a new paper on this subject, by Van Riet et al. [12], which commented on the $10 \mathrm{~d}$ and $4 \mathrm{~d}$ analysis of the KKLT scenario in [11, 16, 17, 42].

With respect to $10 \mathrm{~d}$, the authors of [12] emphasized a problematic issue related to the divergences which appear in the calculations of [11]. However, as we already discussed in section 2 , investigation of this issue in $[11,12]$ missed important terms $[13,14]$. Thus, in agreement with $[15,17]$, we believe that the $10 \mathrm{~d}$ analysis in $[11,12]$ is inconclusive. Moreover, according to [11], their 10d analysis, as well as the one in [12], is model-dependent; it does not apply at all to the version of the KKLT scenario where the moduli stabilization is provided not by gluino condensate but by the Euclidean D-brane instanton effects.

Therefore in the present paper, following [17], we concentrate on the 4d KKLT models. The comments on these models given in [12] are very short and somewhat confusing. We feel that some clarifications are in order.

Ref. [12] makes a conjecture that if the parameter $c$ should be suppressed in order to have a well-defined nilpotent description, then the nilpotent description is not adequate to model the flattening effects, which was the main focus of investigation in [11, 12].

This conjecture consists of two incorrect parts. In the present paper we constructed several consistent $4 \mathrm{~d}$ generalizations of the original KKLT model. These models remain valid for any value of $c$, and they adequately describe the flattening effects, which indeed take place in these models as expected in [11]. Thus there are consistent models where the constant $c$ can be large, and the flattening effect is real. However, as we are going to explain, flattening does not imply the absence of dS vacua, contrary to what was conjectured and many times repeated in $[11,12]$. dS vacua can be found in all of these models, even for extremely large $c$, despite the flattening of the potential.

Strictly speaking, one should not study these issues in the model of [11], as the authors of [12] do, since this model is inconsistent at large $c$. However, as we already mentioned in the previous section, the fully consistent model (3.7) constructed in our paper [17] leads to the same bosonic potential as in [11], in the small $\beta$ limit. In this sense, investigation of the bosonic potential of [11] provides correct information about the uplifting in the consistent model (3.7) at small $\beta$.

However, while it indeed makes sense to study uplifting in the model of [11], the authors of [12] do something different: they instead study destabilization of dS vacua, which occurs after the uplifting, during the further increase of $c$ and $b$. The discussion was based on the figure 2 given in [12], which we reproduce here, with some modifications, using the original notations for the model (3.4), see figure 2 .

The black solid line in figure 2 shows the dS vacuum with a small positive vacuum energy obtained by uplifting with $c=0, b=1.77 \times 10^{-5}$. The dotted lines show further $\mathrm{dS}$ uplift due to increase of $c$ from 0 to $c=0.2, c=0.4, c=0.6$ for $b=1.77 \times 10^{-5}$. As we see, the last increase of $c$ leads to destabilization of the dS minimum. 


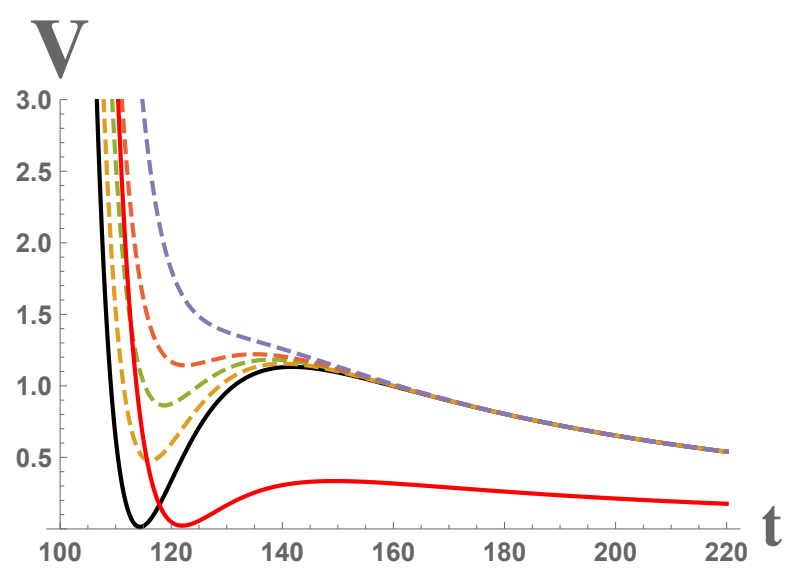

Figure 2. The potential in the model (3.4) (multiplied by $10^{15}$ ) for $A=1, a=0.1, W_{0}=-10^{-4}$. The black line shows the potential at $c=0$ uplifted by increase of $b$ to from 0 to $b=1.77 \times 10^{-5}$. The dotted lines show further uplift due to increase of $c$ from 0 to $c=0.2,0.4,0.6$, and 1 . The last increase of $c$ leads to destabilization of the dS minimum. The red line shows that if one takes $c=1$ and decreases the value of $b$ from $b=1.77 \times 10^{-5}$ to $b=10^{-5}$, one finds a stable dS minimum, the same that is shown by the red line in figure 1 , and in figure 2 in [16].

This clearly shows that there is a large family of $\mathrm{dS}$ vacua in this theory, even for $c$ many orders of magnitude greater than $b$. These results give a simple $4 \mathrm{~d}$ counter-example for the statements of $[11,12]$. Instead of coming to this obvious conclusion, the authors of [12] argue that this scenario is problematic because if one continues increasing $c$ up to $c=1$, this leads to destabilization of the uplifted $\mathrm{dS}$ vacua.

Indeed, it was shown long ago that an excessively strong uplifting leads to dS destabilization [18]. This effect occurs even for $c=0$, if $b$ is too large, so one should choose the uplift parameters carefully [18]. However, this effect has no relation whatsoever to the possibility to uplift from AdS to dS. It is very easy to cure the destabilization of the dS minimum at $c=1, b=1.77 \times 10^{-5}$. It is sufficient to take a slightly smaller value of $b=10^{-5}$, which leads to a stable dS minimum shown by the red line in figure 2 . It is the same red line that is shown in figure 1 in the previous section.

The lesson that follows from this calculation is that the increase of $c$ from 0 to 1 practically does not affect the uplift: the effect of this increase is completely compensated by the less than $50 \%$ decrease of $b$ from $1.77 \times 10^{-5}$ to $10^{-5}$. Moreover, as argued in [17], there is not much reason to even consider the regime with $c \gg b$, because it would imply a physically unreasonable situation where the backreaction to the small impact provided by $b$ is many orders of magnitude stronger than the original impact. We considered the large $c$ regime only to show that even in this case the consistent dS uplift is possible.

But what if $c=\mathcal{O}(1)$, which is 5 orders of magnitude greater than the required value of $b$, still does not seem large enough to convince some authors? To address this question, we now consider an incredibly broad range of values of $c \gg 1$, all the way up to $c=10^{20}$, for $A=1, a=0.1, W_{0}=-10^{-4}$, see figure 3 . To present our results for such a large range of $c \gg 1$ in a single figure, we rescale the potential by multiplying it by $10^{15} c^{2.02} \log c$, 


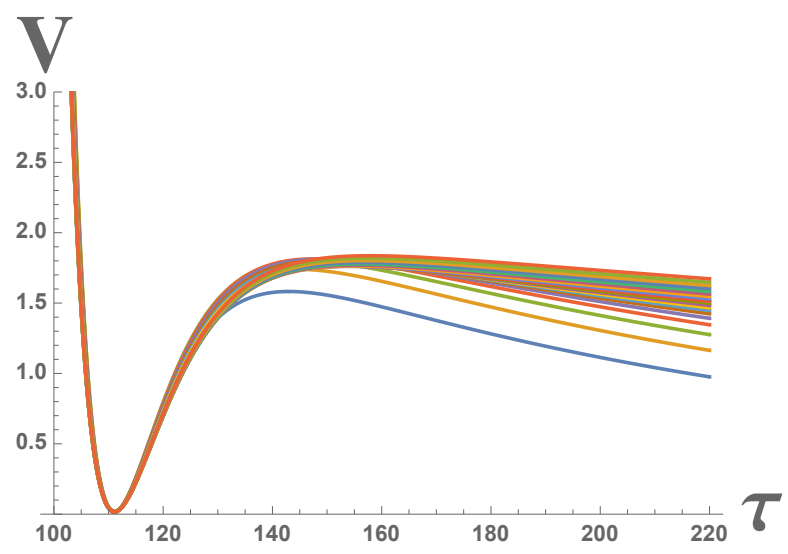

Figure 3. The potential in the model (3.4) multiplied by $10^{15} c^{2.02} \log c$ for $t=\tau+20 \log c$, $b c \approx 1.5 \times 10^{-5}$ and $c$ in the broad range from $c=10^{2}$ to $c=10^{20}$.

and plot it as a function of $\tau=t-20 \log c$. We find a set of dS minima for each value of $c \gg 1$, for $b c \approx 1.5 \times 10^{-5}$. The minima of all of these potentials are at the same point $\tau=t-20 \log c \approx 112$, with the same curvature at the minimum, and approximately the same height of the barrier, making the $\mathrm{dS}$ vacuum metastable.

One may wonder how can one see the promised flattening effect at large $c$ in figure 3? The answer is that the height of the barrier in this figure was enhanced by the factor $\sim c^{2}$ in order to reveal the similarity of the shape of the potential for various $c$. Thus the true height of the barrier decreases approximately as $c^{-2}$ for large $c$, so the flattening effect is very real, even though it occurs only for unreasonably large $c$. But we also see that this effect does not forbid dS vacua.

The opposite statement made in $[11,12,42]$ was based on an appealing but incorrect conjecture that one can ignore $b$ if $c \gg b$. This conjecture was not based on any actual investigation. It is incorrect, because the term proportional to $c$ in (3.4) is exponentially suppressed at large $T=t$. That is why the position of the minimum at large $c$ shifts towards greater values of $t=\tau+20 \log c$, see figure 3. Thus, our results do not reveal any relation between the flattening effect and the existence of dS vacua. In particular, figure 3 demonstrates that well-formed metastable dS minima may exist even if one assumes that $b$ is 45 orders of magnitude smaller than $c$, and the potential is flattened by more than 40 orders of magnitude.

\section{The KL model and the weak gravity conjecture}

A year after the invention of the KKLT model [1], it was recognized that combining this model with inflation effectively leads to an additional contribution to $\mu^{2}$, which could destabilize the volume modulus in the very early universe [18]. The destabilization may occur at a large Hubble constant because the height of the barrier in the KKLT scenario is proportional to the square of $W_{0}$ related to the gravitino mass and the strength of supersymmetry breaking, which was often considered small. 
This problem disappears if supersymmetry breaking in this theory is sufficiently high, but there are several other ways to stabilize the KKLT potential. The simplest one, proposed in [18], is to change the superpotential to the racetrack potential with two exponents,

$$
W_{\mathrm{KL}}(T, S)=W_{0}+A e^{-a T}-B e^{-b T}+\mu^{2} S,
$$

which can arise, for example, in the presence of two stacks of D7 branes wrapping homologous 4-cycles. Gaugino condensation on the first one is responsible for the KKLT-type term $A e^{-a T}$, the second one for the term $-B e^{-b T}$. If there are $N_{1}$ branes in the first stack, and $N_{2}$ branes in the second one, one has $a=2 \pi / N_{1}$ and $b=2 \pi / N_{2}$. We assume, without loss of generality, that $a>b$. The parameters $A$ and $B$ depend on the values at which the complex structure moduli are stabilized [29, 43, 44], and therefore one may expect $A$ and $B$ to span large range of possible values, due to the large variety of vacua in the string theory landscape.

In what follows we will consider the models where $a, b, A, B>0$, and

$$
W_{0}=-A\left(\frac{a A}{b B}\right)^{\frac{a}{b-a}}+B\left(\frac{a A}{b B}\right)^{\frac{b}{b-a}} .
$$

For $\mu=0$, the potential $V(T)$ has a stable supersymmetric Minkowski minimum at $T=t_{0}$, which can be found by solving two equations,

$$
W\left(t_{0}\right)=0, \quad D W\left(t_{0}\right)=0,
$$

which yields

$$
t_{0}=\frac{1}{a-b} \ln \frac{a A}{b B} .
$$

Adding a small correction to $W_{0}$ makes this minimum AdS. For $\mu \neq 0$, this minimum can be easily uplifted to dS while remaining strongly stabilized [18, 21, 22]. Importantly, the height of the barrier in this scenario is not related to supersymmetry breaking and can be arbitrarily high. Therefore, this potential can be strongly stabilized by a proper choice of the parameters, which makes it especially suitable for being a part of the inflationary theory [23-25].

The KL model (5.1) with the racetrack superpotential is just one of the many possible ways to find nearly supersymmetric vacua. One may consider general superpotentials which may emerge among $10^{500}$ or more versions of string theory compactification, after all quantum corrections are taken into account. If there is a point in the moduli space where supersymmetry is unbroken in Minkowski space (e.g. where equations (5.3) have a solution), then the tiny uplift required for describing dS space with an incredibly small cosmological constant $\sim 10^{-120}$ does not lead to vacuum destabilization [21, 22]. We should emphasize that for our purposes (the theory of vacuum stabilization) we do not need to require flatness of the potential, large excursions of the field away from the vacuum state, etc. We only need to make sure that the depth of the AdS (nearly Minkowski) minimum is parametrically smaller than the squares of the masses of all moduli in the vicinity of the minimum. This can be achieved in the KL construction. 
If the number of possible vacua in the landscape is large enough, the number of nearly supersymmetric vacua should be also extremely large, which should address the general problem of uplifting in such vacua. However, it is always nice to have explicit examples of the models where it may happen in a controllable way.

The status of such models can be probed by applying simple consistency tests. Just like in the original version of the KKLT scenario, one should check that the radius of compactification $t_{0}$ sufficiently large, which is necessary for consistency of the supergravity interpretation. One can achieve it, for any $A$ and $B$, by considering sufficiently small $a$ and $b$.

In analogy with axion scalar potential generated by instantons, and with the knowledge that D-brane instantons enter the theory via the superpotential $[45,46]$, one might anticipate higher order non-perturbative contributions to the gaugino condensate superpotentials in the KL model. The precise sense in which this intuition from instantons can be applied to gaugino condensation is via the dual description of gaugino condensation as Euclidean M5 brane instantons in M-theory (see e.g. [47] for a detailed technical discussion of related issues). Putting aside the technical details of the origin of such terms, one would expect that higher order non-perturbative contributions to the gaugino condensate superpotential should be under control near the minimum of the KL potential if

$$
e^{-a t_{0}}<1, \quad e^{-b t_{0}}<1 .
$$

Note that the terms $e^{-a t_{0}}$ and $e^{-b t_{0}}$ are strongly suppressed even for moderately large values of $a t_{0}$ and $b t_{0}$,

$$
a t_{0}>1, \quad b t_{0}>1 .
$$

Using (5.4), we can represent the conditions (5.5) as follows:

$$
e^{-a t_{0}}=\left(\frac{b B}{a A}\right)^{\frac{a}{a-b}}<1, \quad e^{-b t_{0}}=\left(\frac{b B}{a A}\right)^{\frac{b}{a-b}}<1 .
$$

All these conditions are satisfied, for example, if $b \gtrsim a / 2$ and $A \gtrsim 2 B$. Thus the KL model is expected to be consistent and reliable in the vicinity of the minimum of its potential $t_{0}$ for a broad choice of parameters.

Consider, for example, the case $b=a / 2$. Then one can check that the strongest constraint originates from the second inequality,

$$
e^{-b t_{0}}=\frac{B}{2 A}<1
$$

This expansion parameter is smaller than $1 / 4$ for $A>2 B$, and is smaller than $10^{-1}$ for $A>5 B$. It becomes even easier to satisfy all conditions mentioned above for $a-b \ll a$.

Then where does the statement of Moritz and Van Riet [19] that the KL model may contradict the weak gravity conjecture come from?

To understand it, let us discuss the relation between the conditions of the type of (5.5), (5.7) and the weak gravity conjecture for the simplest axion inflation models with the Higgs-type potential $\sim\left(|\phi|^{2}-f_{a}^{2}\right)^{2}$, as in natural inflation. For such axions, there 
is a series of instantons with action $S_{n}=O\left(n S_{1}\right)$, where $n=1,2, \ldots$, see e.g. [48]. Here $f_{a}$ is the axion decay constant, which describes, up to a factor $2 \pi$, the periodicity of the axion potential. One can trust the axion potential ignoring higher order instanton corrections $\sim e^{-n S_{1}}$ in the natural inflation scenario only if $e^{-S_{1}}<1$, i.e. if $f_{a}<1$. This is the condition that makes natural inflation so difficult to implement.

On the other hand, if, like in the string theory landscape, we do not care about natural inflation, about quantum corrections and the shape of the potential in the angular direction, and only want to make sure that the potential has some minimum somewhere, then the constraint $e^{-S_{1}}<1$ becomes less relevant.

Now let us return for a moment to the KKLT scenario with a superpotential with a single exponent $W=W_{0}-A e^{-a T}+b S$ (3.1). The requirement of the exponentially large suppression of nonperturbative effects in a vicinity of the minimum of the KKLT potential requires the condition $e^{-a t_{0}}<1$, with $t_{0}$ being the position of the minimum. Just as in the natural inflation scenario, this requirement is equivalent, up to a factor $\sqrt{3 / 2}$, to the weak gravity conjecture requirement that the effective axion decay constant $f_{a} \lesssim 1$, where $f_{a}$ describes the periodicity of the KKLT potential in the $\operatorname{Im} T$ direction in canonical variables $\theta=\sqrt{\frac{2}{3}} \frac{\alpha}{t_{0}}$ for $\operatorname{Re} T=t_{0}[19]$. Thus, just as in the usual axion scenario, the smallness of the effective axion decay constant $f_{a}$ in the KKLT model appears as a consequence of the requirement that the higher order nonperturbative effects are suppressed, $e^{-a t_{0}}<1$.

However, already at this level the situation is somewhat different from the one in natural inflation. First of all, we are unaware of any higher order KKLT instantons with $S_{n}=O\left(n / f_{a}\right)$ for $n>1$. Therefore, while the requirement that higher order nonperturbative corrections should be suppressed seems reasonable, at this stage it is somewhat speculative. Secondly, there is no inflation in the axionic direction in the KKLT model, so we do not need to know the exact form of the KKLT potential in the axion direction. As we already discussed, the main requirement is that it should have a dS minimum somewhere, after potentially significant quantum corrections are taken into account. However, it is important to have $t_{0} \gg 1$, to ensure that the supergravity approach to string theory is adequate. To be on the safe side, one may also impose the condition $a t_{0}>1$. One can easily find parameters which satisfy both of these conditions, but in general, the condition $a t_{0}>1$ may or may not be required for the existence of dS vacua in KKLT.

Note that the most important features of the KKLT potential, such as the position of the AdS minimum, the position of the dS minimum after uplifting, and the potential barrier responsible for the vacuum stabilization are concentrated at $t \sim t_{0}$. Therefore all consistency constraints mentioned above are imposed on the theory at $t \sim t_{0}$. The theory may become unreliable for $t \ll t_{0}$, and indeed we do not expect that the supergravity approximation is adequate for $t \lesssim 1$, but this is not our immediate concern as long as $t_{0} \gg 1$.

Finally, we return to the KL scenario. The full potential as a function of $T=t+i \alpha$ is

$$
\begin{aligned}
V_{\mathrm{KL}}= & \frac{1}{6 t^{2}}\left(a A^{2} e^{-2 a t}(a t+3)+b B^{2} e^{-2 b t}(b t+3)\right. \\
& +3 a A W_{0} e^{-a t} \cos a \alpha-3 b B W_{0} e^{-b t} \cos b \alpha \\
& \left.-A B e^{-(a+b) t}(3(a+b)+2 a b t) \cos (a-b) \alpha\right) .
\end{aligned}
$$


Despite the fact that we consider a single axion field $\alpha$, we see that the potential has three different periodicities along the axion direction. One may try to describe this property of the potential by introducing effective axion decay constants $f_{a} \sim 1 / a t_{0}, f_{b} \sim 1 / b t_{0}$, and $f_{a-b} \sim 1 /(a-b) t_{0}$. However, this interpretation can be misleading.

Indeed, nonperturbative terms in the KL superpotential (5.1) are exponentially suppressed for $f_{a}^{-1}>1, f_{b}^{-1}>1$ (5.7). It could be tempting, following [19], to introduce an additional condition

$$
f_{a-b}^{-1} \sim(a-b) t_{0}>1
$$

similar to the conditions (5.6), or require that the corresponding instanton-type expansion parameter is small, $e^{-(a-b) t_{0}}<1$, as in (5.5).

But it is hard to justify this additional requirement. Indeed, if the expression for the superpotential (5.1) is reliable because of the exponential suppression of the nonperturbative terms, then the expression for the last term in (5.9) is also reliable. No higher order effects suppressed by $e^{-(a-b) t_{0}}$ are known to us. Thus, in our opinion, the consistency conditions (5.5) should be sufficient, and until one provides a good reason for an additional constraint (5.10), one may simply ignore it.

However, suppose that one wants to err on the side of caution and take the speculative condition (5.10) seriously. As we will see, this is not a problem as well. The problem discussed in [19] appears only if one makes two other steps. First of all, the authors of [19] assumed that $a-b \ll a$, which is not required in the KL model; for example, it was used in [18], but not in [23]. Secondly, the authors of [19] imposed an additional condition that the last term in (5.9) must be smaller than the two previous terms for $t \sim 1 / a \approx 1 / b$.

In our opinion, this last step is unwarranted. Indeed, in the limit $a-b \ll a$ the conditions (5.5) imply that $t \sim 1 / a \approx 1 / b \ll t_{0}$. Thus the authors of [19] imposed their conditions at $t \ll t_{0}$, at the steep potential wall, very far away from the minimum. This led them to a strong additional constraint $\left|W_{0}\right| \gtrsim \min (|\mathrm{A}|,|\mathrm{B}|)$ (eq. (16) of [19]), which played the central role in their investigation, but which is not actually required for the consistency of the KL model.

The main requirement for any model of $\mathrm{dS}$ vacuum stabilization in string theory is to be valid in the vicinity of the $\mathrm{dS}$ minimum of the potential for some range of values $t=\mathcal{O}\left(t_{0}\right)$, see e.g. [49]. That is exactly what we did formulating the conditions (5.5), and even the speculative condition (5.10). All important features of the KL potential appear at $t \gtrsim t_{0}$. If the consistency conditions (5.5) and (5.10) are satisfied at $t \sim t_{0}$, then they are automatically satisfied at all $t \gtrsim t_{0}$.

By using (5.4), one finds that for $t=\mathcal{O}\left(t_{0}\right)$ the coefficient $(a-b)$ in (5.10) is canceled by the pole $1 /(a-b)$ in (5.4), and the strong form of the weak gravity conjecture (5.10) proposed in [19] is satisfied for a broad range of parameters such that

$$
f_{a-b}^{-1} \sim \ln \frac{a A}{b B}>1 .
$$

According to the discussion of the racetrack potential in eq. (21) of [43],

$$
\left|\frac{a A}{b B}\right|=\left(\frac{\tilde{\mu}_{1}}{\tilde{\mu}_{2}}\right)^{3} \text {. }
$$


Here $a=2 \pi / N_{1}, b=2 \pi / N_{2}, A=N_{1} \tilde{\mu}_{1}^{3}, B=N_{2} \tilde{\mu}_{2}^{3}$. There are no known restrictions on the size or sign of the ratio $\tilde{\mu}_{2} / \tilde{\mu}_{1}$. These quantities depend on the values of the complex structure moduli, as discussed in [29, 44]. They may take different values for each stack of the branes, depending on moduli stabilization.

To give some particular examples, let us first consider the case $b=a / 2$. In this case, one has $(a-b) t=b t$, therefore the additional constraint (5.10) introduced in [19] exactly coincides with our second constraint in (5.6). Therefore for $b=a / 2$ the weak gravity conjecture proposed in [19] does not lead to any additional constraints on the parameters of the KL model. One can show that all required conditions, including $a t_{0}>1, b t_{0}>1$, and $(a-b) t_{0}>1$, are satisfied for $A \gtrsim 2 B$. Our results are consistent with the results of ref. [20]. Similar results are valid as well for the more general case $a>b \gtrsim a / 2$ (including the case $a-b \ll a)$ and $A \gtrsim 3 B$. We are unaware of any no-go theorems that would forbid having such parameters in the string theory landscape.

We conclude, that for a broad choice of parameters, the KL version of the KKLT construction does not violate the standard consistency constraints (5.5), and even the speculative form of the weak gravity conjecture (5.10) introduced in [19].

\section{Conclusions}

In this paper we note that the $10 \mathrm{~d}$ arguments of $[11,12]$ against $\mathrm{dS}$ uplifting were based on a number of conjectures and an incomplete theory of gaugino condensation, missing important terms $[13,14]$. The full $10 \mathrm{~d}$ theory is expected to provide results compatible with the $4 \mathrm{~d}$ description of the KKLT scenario. All presently available consistent generalizations of the $4 \mathrm{~d}$ KKLT model discussed in $[16,17]$ and in the present paper reveal the existence of dS vacua in the KKLT scenario.

We also argue that the KL version of the KKLT scenario [18], which is based on a racetrack superpotential and does not present any problems with uplifting [11], is compatible with the weak gravity conjecture for a broad choice of parameters of the KL model.

Thus, the results of our analysis of all presently available consistent generalizations of the $4 \mathrm{~d}$ KKLT model do not support the recent swampland conjecture $[6,7]$ and are fully compatible with the existence of $\mathrm{dS}$ vacua in string theory.

\section{Acknowledgments}

We would like to thank Sergio Ferrara, Shamit Kachru, Liam McAllister, Miguel Montero, Jakob Moritz, Susha Parameswaran, Tom Rudelius, Eva Silverstein, Sandip Trivedi, Vincent Van Hemelryck, Thomas Van Riet, Alexander Westphal, and Timm Wrase for helpful comments and discussions. The work of RK and AL is supported by SITP, by the NSF Grant PHY-1720397, and by the Simons Foundation grant. EM is supported in part by the National Science and Engineering Research Council of Canada via a PDF fellowship. MS is supported by the Research Foundation - Flanders (FWO) and the European Union's Horizon 2020 research and innovation programme under the Marie Skłodowska-Curie grant agreement No. 665501. 
Open Access. This article is distributed under the terms of the Creative Commons Attribution License (CC-BY 4.0), which permits any use, distribution and reproduction in any medium, provided the original author(s) and source are credited.

\section{References}

[1] S. Kachru, R. Kallosh, A.D. Linde and S.P. Trivedi, de Sitter vacua in string theory, Phys. Rev. D 68 (2003) 046005 [hep-th/0301240] [INSPIRE].

[2] M.R. Douglas, The Statistics of string/M theory vacua, JHEP 05 (2003) 046 [hep-th/0303194] [INSPIRE].

[3] M.R. Douglas and S. Kachru, Flux compactification, Rev. Mod. Phys. 79 (2007) 733 [hep-th/0610102] [INSPIRE].

[4] F. Denef, M.R. Douglas and S. Kachru, Physics of String Flux Compactifications, Ann. Rev. Nucl. Part. Sci. 57 (2007) 119 [hep-th/0701050] [INSPIRE].

[5] L. Susskind, The Anthropic landscape of string theory, hep-th/0302219 [INSPIRE].

[6] G. Obied, H. Ooguri, L. Spodyneiko and C. Vafa, de Sitter Space and the Swampland, arXiv: 1806.08362 [INSPIRE].

[7] H. Ooguri, E. Palti, G. Shiu and C. Vafa, Distance and de Sitter Conjectures on the Swampland, Phys. Lett. B 788 (2019) 180 [arXiv:1810.05506] [InSPIRE].

[8] Y. Akrami, R. Kallosh, A. Linde and V. Vardanyan, The Landscape, the Swampland and the Era of Precision Cosmology, Fortsch. Phys. 67 (2019) 1800075 [arXiv: 1808. 09440] [INSPIRE].

[9] M. Raveri, W. Hu and S. Sethi, Swampland Conjectures and Late-Time Cosmology, arXiv: 1812.10448 [INSPIRE].

[10] Y. Akrami, R. Kallosh, A. Linde and V. Vardanyan, Dark energy, $\alpha$-attractors and large-scale structure surveys, JCAP 06 (2018) 041 [arXiv: 1712.09693] [INSPIRE].

[11] J. Moritz, A. Retolaza and A. Westphal, Toward de Sitter space from ten dimensions, Phys. Rev. D 97 (2018) 046010 [arXiv:1707.08678] [InSPIRE].

[12] F.F. Gautason, V. Van Hemelryck and T. Van Riet, The Tension between $10 D$ Supergravity and dS Uplifts, Fortsch. Phys. 67 (2019) 1800091 [arXiv:1810.08518] [INSPIRE].

[13] Y. Hamada, A. Hebecker, G. Shiu and P. Soler, Brane gaugino condensate in 10d, arXiv: 1812.06097 [INSPIRE].

[14] R. Kallosh, Gaugino Condensation and Geometry of the Perfect Square, Phys. Rev. D 99 (2019) 066003 [arXiv:1901.02023] [INSPIRE].

[15] M. Cicoli, S. De Alwis, A. Maharana, F. Muia and F. Quevedo, de Sitter vs Quintessence in String Theory, Fortsch. Phys. 67 (2019) 1800079 [arXiv:1808.08967] [INSPIRE].

[16] R. Kallosh, A. Linde, E. McDonough and M. Scalisi, de Sitter Vacua with a Nilpotent Superfield, Fortsch. Phys. 67 (2019) 1800068 [arXiv: 1808.09428] [INSPIRE].

[17] R. Kallosh, A. Linde, E. McDonough and M. Scalisi, $4 D$ models of de Sitter uplift, Phys. Rev. D 99 (2019) 046006 [arXiv:1809.09018] [INSPIRE].

[18] R. Kallosh and A.D. Linde, Landscape, the scale of SUSY breaking and inflation, JHEP 12 (2004) 004 [hep-th/0411011] [inSPIRE]. 
[19] J. Moritz and T. Van Riet, Racing through the swampland: de Sitter uplift vs weak gravity, JHEP 09 (2018) 099 [arXiv: 1805.00944] [INSPIRE].

[20] J.J. Blanco-Pillado, M.A. Urkiola and J.M. Wachter, Racetrack Potentials and the de Sitter Swampland Conjectures, JHEP 01 (2019) 187 [arXiv:1811.05463] [INSPIRE].

[21] J.J. Blanco-Pillado, R. Kallosh and A.D. Linde, Supersymmetry and stability of flux vacua, JHEP 05 (2006) 053 [hep-th/0511042] [INSPIRE].

[22] R. Kallosh, A. Linde, B. Vercnocke and T. Wrase, Analytic Classes of Metastable de Sitter Vacua, JHEP 10 (2014) 011 [arXiv: 1406.4866] [INSPIRE].

[23] R. Kallosh, A. Linde, K.A. Olive and T. Rube, Chaotic inflation and supersymmetry breaking, Phys. Rev. D 84 (2011) 083519 [arXiv:1106.6025] [InSPIRE].

[24] E. Dudas, A. Linde, Y. Mambrini, A. Mustafayev and K.A. Olive, Strong moduli stabilization and phenomenology, Eur. Phys. J. C 73 (2013) 2268 [arXiv:1209.0499] [InSPIRE].

[25] R. Kallosh, A. Linde and Y. Yamada, Planck 2018 and Brane Inflation Revisited, JHEP 01 (2019) 008 [arXiv: 1811.01023] [INSPIRE].

[26] D. Marolf, L. Martucci and P.J. Silva, Fermions, T duality and effective actions for D-branes in bosonic backgrounds, JHEP 04 (2003) 051 [hep-th/0303209] [INSPIRE].

[27] P.G. Camara, L.E. Ibáñez and A.M. Uranga, Flux-induced SUSY-breaking soft terms on D\%-D3 brane systems, Nucl. Phys. B 708 (2005) 268 [hep-th/0408036] [INSPIRE].

[28] L. Martucci, J. Rosseel, D. Van den Bleeken and A. Van Proeyen, Dirac actions for D-branes on backgrounds with fluxes, Class. Quant. Grav. 22 (2005) 2745 [hep-th/0504041] [INSPIRE].

[29] D. Baumann, A. Dymarsky, S. Kachru, I.R. Klebanov and L. McAllister, D3-brane Potentials from Fluxes in AdS/CFT, JHEP 06 (2010) 072 [arXiv:1001.5028] [INSPIRE].

[30] A. Dymarsky and L. Martucci, D-brane non-perturbative effects and geometric deformations, JHEP 04 (2011) 061 [arXiv: 1012.4018] [INSPIRE].

[31] P. Hořava and E. Witten, Heterotic and type-I string dynamics from eleven-dimensions, Nucl. Phys. B 460 (1996) 506 [hep-th/9510209] [INSPIRE].

[32] P. Hořava and E. Witten, Eleven-dimensional supergravity on a manifold with boundary, Nucl. Phys. B 475 (1996) 94 [hep-th/9603142] [INSPIRE].

[33] P. Hořava, Gluino condensation in strongly coupled heterotic string theory, Phys. Rev. D 54 (1996) 7561 [hep-th/9608019] [INSPIRE].

[34] S. Ferrara, L. Girardello and H.P. Nilles, Breakdown of Local Supersymmetry Through Gauge Fermion Condensates, Phys. Lett. B 125 (1983) 457 [InSPIRE].

[35] E. Cremmer, S. Ferrara, L. Girardello and A. Van Proeyen, Yang-Mills Theories with Local Supersymmetry: Lagrangian, Transformation Laws and SuperHiggs Effect, Nucl. Phys. B 212 (1983) 413 [INSPIRE].

[36] E. Bergshoeff, M. de Roo, B. de Wit and P. van Nieuwenhuizen, Ten-Dimensional Maxwell-Einstein Supergravity, Its Currents and the Issue of Its Auxiliary Fields, Nucl. Phys. B 195 (1982) 97 [INSPIRE].

[37] G.F. Chapline and N.S. Manton, Unification of Yang-Mills Theory and Supergravity in Ten-Dimensions, Phys. Lett. B 120 (1983) 105 [InSPIRE]. 
[38] M. Dine, R. Rohm, N. Seiberg and E. Witten, Gluino Condensation in Superstring Models, Phys. Lett. B 156 (1985) 55 [INSPIRE].

[39] S. Ferrara, R. Kallosh and A. Linde, Cosmology with Nilpotent Superfields, JHEP 10 (2014) 143 [arXiv: 1408.4096] [INSPIRE].

[40] R. Kallosh and T. Wrase, Emergence of Spontaneously Broken Supersymmetry on an Anti-D3-Brane in KKLT dS Vacua, JHEP 12 (2014) 117 [arXiv:1411.1121] [INSPIRE].

[41] E.A. Bergshoeff, K. Dasgupta, R. Kallosh, A. Van Proeyen and T. Wrase, $\overline{\mathrm{D} 3}$ and $d S, J H E P$ 05 (2015) 058 [arXiv: 1502.07627] [INSPIRE].

[42] J. Moritz, A. Retolaza and A. Westphal, On uplifts by warped anti-D3-branes, Fortsch. Phys. 67 (2019) 1800098 [arXiv: 1809.06618] [INSPIRE].

[43] C.P. Burgess, A. de la Macorra, I. Maksymyk and F. Quevedo, Supersymmetric models with product groups and field dependent gauge couplings, JHEP 09 (1998) 007 [hep-th/9808087] [INSPIRE].

[44] D. Baumann, A. Dymarsky, I.R. Klebanov, J.M. Maldacena, L.P. McAllister and A. Murugan, On D3-brane Potentials in Compactifications with Fluxes and Wrapped D-branes, JHEP 11 (2006) 031 [hep-th/0607050] [INSPIRE].

[45] E. Witten, Nonperturbative superpotentials in string theory, Nucl. Phys. B 474 (1996) 343 [hep-th/9604030] [INSPIRE].

[46] J.A. Harvey and G.W. Moore, Superpotentials and membrane instantons, hep-th/9907026 [INSPIRE].

[47] F. Denef, Les Houches Lectures on Constructing String Vacua, Les Houches 87 (2008) 483 [arXiv:0803.1194] [INSPIRE].

[48] R. Blumenhagen, Large Field Inflation/Quintessence and the Refined Swampland Distance Conjecture, PoS (CORFU2017) 175 [arXiv: 1804 .10504] [INSPIRE].

[49] D. Baumann and L. McAllister, Inflation and String Theory, Cambridge Monographs on Mathematical Physics, Cambridge University Press, Cambridge U.K. (2015) http://www.cambridge.org/mw/academic/subjects/physics/theoretical-physics-andmathematical-physics/inflation-and-string-theory?format $=\mathrm{HB}$ [arXiv:1404.2601] [INSPIRE]. 